\title{
Analytical derivation: An epistemic game for solving mathematically based physics problems
}

\author{
Rabindra R. Bajracharya ${ }^{1, *}$ and John R. Thompson ${ }^{2,3, \dagger}$ \\ ${ }^{1}$ Department of Physics, 301 Weniger Hall, Oregon State University, Corvallis, Oregon 97331, USA \\ ${ }^{2}$ Department of Physics and Astronomy, University of Maine, \\ 5709 Bennett Hall, Orono, Maine 04469-5709, USA \\ ${ }^{3}$ Maine Center for Research in STEM Education, University of Maine, \\ 5727 Estabrooke Hall, Orono, Maine 04469-5727, USA
}

(Received 24 July 2015; published 30 March 2016)

\begin{abstract}
Problem solving, which often involves multiple steps, is an integral part of physics learning and teaching. Using the perspective of the epistemic game, we documented a specific game that is commonly pursued by students while solving mathematically based physics problems: the analytical derivation game. This game involves deriving an equation through symbolic manipulations and routine mathematical operations, usually without any physical interpretation of the processes. This game often creates cognitive obstacles in students, preventing them from using alternative resources or better approaches during problem solving. We conducted hour-long, semi-structured, individual interviews with fourteen introductory physics students. Students were asked to solve four "pseudophysics" problems containing algebraic and graphical representations. The problems required the application of the fundamental theorem of calculus (FTC), which is one of the most frequently used mathematical concepts in physics problem solving. We show that the analytical derivation game is necessary, but not sufficient, to solve mathematically based physics problems, specifically those involving graphical representations.
\end{abstract}

DOI: 10.1103/PhysRevPhysEducRes.12.010124

\section{INTRODUCTION}

One of the most prevailing and widely accepted objectives of physics education is promoting students' problemsolving skills. A student's strategy for solving a physics problem is often based on his or her prior knowledge and patterns of his or her reasoning. In physics, problems are often presented as situations that require resolution. Generally, the resolutions are not trivial and require the use of some thinking process(es), skills, prior knowledge, and strategies.

Mathematics is an important aspect of physics problem solving. Students often learn most of the mathematical concepts and skills that are required to solve physics problems in their mathematics classes. However, numerous studies suggest that they fail to access and/or apply their previously acquired mathematics knowledge in given physics contexts [1-4]. Previous studies have established various factors that contribute to this failure, such as representational inconsistencies between mathematics

\footnotetext{
*bajracharya-r@mssu.edu

Present address: Chemical and Physical Sciences Department, Missouri Southern State University, 3950 Newman Road, Joplin, MO 64801-1595, USA.

thompsonj@maine.edu

Published by the American Physical Society under the terms of the Creative Commons Attribution 3.0 License. Further distribution of this work must maintain attribution to the author(s) and the published article's title, journal citation, and DOI.
}

and physics. Students perceive two mathematically analogous problems completely differently when the problems are presented in physics and mathematical contexts, respectively $[1,5,6]$. Problem solving in physics often requires identification of the relationships between the physics and the mathematics that are built into the representation as well as subsequent interpretation and application of the related mathematical concepts $[5,7,8]$. Mathematics provides logical paths and methods to solve complex physics problems. Thus, students are expected to have a good grasp of at least basic mathematical skills in order for them to be able to apply these skills during problem solving. There has been little research on the mathematical aspect of physics problem solving [5,9-11]. Previous studies have shown a positive correlation between student difficulties with physics concepts and those with either the mathematics concepts, application of those concepts, or the representations used to connect the mathematics and the physics. Students lacking and/or failing to apply relevant mathematical knowledge and skills have shown difficulties with reasoning and/or solving problems in physics contexts $[2,3,12,13]$.

A few models have been proposed in physics education research to explain the properties of a normative problem solving process. Redish proposed a four-step model for solving a physics problem [5]. The four steps include (i) modeling the physical system to mathematical representation, (ii) processing the mathematical operations, (iii) interpreting the results of the mathematical operations in terms of the physical system, and (iv) evaluating the 
results to justify the initial modeling. Wilcox and colleagues refined Redish's model to explain students'use of mathematics in upper-division physics [10]. Their framework also contains four elements in the problem-solving process including activation of mathematical tools, construction of mathematical models, execution of the mathematics, and reflection on the results (ACER).

As a part of our ongoing disciplinary-based education research program, we have been studying student interpretation and application of graphical representations at the mathematics-physics interface. In this study, we investigated student strategies for solving physics problems with graphical representations that require the use of the fundamental theorem of calculus (FTC). In addition, we also studied how students invoked and applied their mathematical knowledge and skills while solving the problems. The complexity of the physical contexts of the problems was reduced as much as possible to keep the students focused on the mathematical aspects, and to make their application of mathematics more apparent. However, the physics contexts we used were chosen for their familiarity in instructional situations. Because our intent was to study student problem-solving behavior, we found the perspectives of epistemological framing and epistemic games to be helpful and relevant lenses through which to analyze and interpret the observed student problem-solving approaches. Our analysis led us to propose a new epistemic game that was commonly observed in this study-the analytical derivation game. We establish the parameters for analytical derivation and provide evidence for those parameters. We also show how analytical derivation is different from existing, seemingly similar epistemic games, and how students enter and exit the game from other existing games. Finally, we argue that analytical derivation is a broad, large-scale epistemic game that is played by students in many problem-solving tasks.

\section{BACKGROUND}

In this section, we present a brief review on the relevance of the FTC in physics problem solving, previous studies on student understanding of the FTC and the definite integral in physics and mathematics education, and the epistemic game and epistemological framing frameworks and their relevance to this study.

\section{A. Student understanding of the fundamental theorem of calculus}

The fundamental theorem of calculus is one of the most frequently applied mathematical tools in physics reasoning and/or problem solving. The FTC has the following two parts, typically referred to as the first and second fundamental theorems [Eqs. (1) and (2)]. The first FTC states that if a function $f(x)$ is continuous on $[a, b]$, then $\int_{a}^{x} f(t) d t$ is differentiable on $(a, b)$ and

$$
\frac{d}{d x} \int_{a}^{x} f(t) d t=f(x) .
$$

The second FTC states that if $F(x)$ is an antiderivative of $f(x)$, that is, $F^{\prime}(x)=f(x)$, then

$$
\int_{a}^{b} f(x) d x=F(b)-F(a) .
$$

Although a few studies in undergraduate mathematics education have explicitly explored student understanding of and difficulties with FTC concepts, they do not attend to specific aspects of the FTC that may be important to physics problem solving, such as the specific form of the representation and the context $[14,15]$. Most of the research in undergraduate mathematics education on the FTC focuses on the integral function [Eq. (1), the first FTC], whereas most physics problems require finding specific values using a definite integral [Eq. (2), the second FTC], in particular physical situations. For example, defining the electric field in one dimension as the negative rate of change of electric potential with respect to position, one can write the potential difference between two any points as

$$
V(b)-V(a)=-\int_{a}^{b} \vec{E}(\vec{r}) \cdot \vec{d} r,
$$

where

$$
E(r)=-\frac{d V}{d r} .
$$

Because this study focused exclusively on the physical application of the relationship between the definite integral and the antiderivative difference as in Eq. (3), we use the term "FTC" to refer the second fundamental theorem of calculus.

Despite its relevance to a wide range of physics content, e.g., kinematics, dynamics, thermodynamics, and quantum mechanics, there have not been explicit studies on student application of the FTC (except a few on definite integrals) in physics education that probe students' abilities to connect the definite integral, the antiderivative difference, and the area under the curve, and to apply them in physics contexts. A few studies in PER implicitly indicate that students have difficulties with various facets of the FTC, such as the definite integral and its graphical representation (area under the curve) in physics contexts $[16,17]$.

Beichner showed implicitly that student have difficulties connecting two important facets of the second FTC, namely, the difference of a physical quantity at two given points [e.g., $\left.v\left(t_{2}\right)-v\left(t_{1}\right)\right]$ and the graph of its rate (e.g., $a-t$ ) [16]. Similarly, our own previous study on student interpretation of the signs of definite integrals showed that students inappropriately used the FTC [i.e., $\int_{a}^{b} f(x) d x=$ $f(b)-f(a)$ ] to determine the signs of integrals [18]. Previously, we identified a number of difficulties that 
students have with understanding and applying the FTC [19]. However, deeper questions emerged as we analyzed student problem-solving behavior, such as (a) What are the origins of the observed difficulties? (b) How deeply are they rooted? and (c) How do the students' difficulties affect their problem-solving strategies? While analyzing student problem-solving strategies for the FTC-based physics problems in graphical representations, we found that students commonly used one particular strategy even when the strategy did not result in an adequate answer. We analyzed this particular strategy deeply using the perspective of epistemic games.

\section{B. Epistemic games}

Epistemic games are the generalization of problemsolving processes directed by a targeted outcome. The framework originated in the field of the learning sciences. According to Collins and Ferguson [20], epistemic games are sets of rules and strategies that are guided by a specific purpose, e.g., learning a concept. Depending on his or her pre-existing knowledge, an individual may use, and frequently switch among, various strategies while solving a problem, until he or she obtains a desired result. Collins and Ferguson proposed three general types of epistemic games: structural analysis, functional analysis, and process analysis. The purpose of structural analysis games is to discover the components or elements of a system. For example, the list making game, which is a type of structural analysis game, involves identifying a task and its target entities, making a list of target entities, combining two or more of the entities, removing unwanted entities, and forming a final list. The objective of functional analysis games is to demonstrate how the elements in a system are associated with each other, e.g., making a causal-chain diagram or creating a hierarchical chart. Process analysis games are intended to illustrate the behavior of a system, e.g., graphing the change in a system over time or drawing a program flowchart.

Tuminaro and Redish adopted the epistemic games perspective in physics education research to explain student strategies for solving algebra-based physics problems [9]. Unlike Collins and Ferguson's normative (expert-based) epistemic games, Tuminaro and Redish used this notion to account for ordinary students' (nonexperts') problemsolving strategies. They classified individual epistemic games as comprised of four components: epistemic form, knowledge base, entry and exit conditions, and moves. The epistemic form is the target structure that helps to guide inquiry during a problem-solving process. A periodic table, squares in a tic-tac-toe game, a flow chart of an algorithm, equations, maps, etc. are a few examples of epistemic forms. The knowledge base in an epistemic game (e-game) is the collection of all the resources (content knowledge, concepts, mathematical skills, epistemology, etc.) that an individual uses during the process. The entry and exit

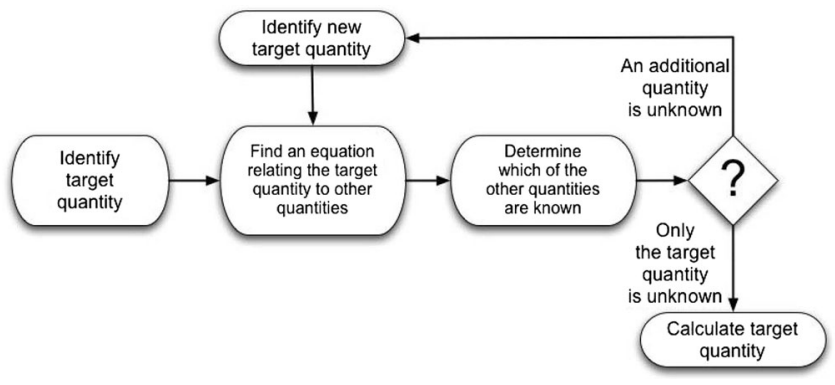

FIG. 1. Tuminaro's schematic diagram for the recursive plugand-chug epistemic game. From Ref. [9].

conditions are the conditions that an individual recognizes to begin and end a particular e-game. The entry condition is often guided by the individual's initial framing of the problem, e.g., conceptual versus analytical. The moves in a game consist of all the steps the individual undertakes during the problem-solving process. Tuminaro and Redish identified and characterized six types of epistemic games for students' application of mathematics in algebra-based physics contexts: (a) pictorial analysis, (b) physical mechanism, (c) recursive plug-and-chug, (d) transliteration to mathematics, (e) mapping [from] meaning to mathematics, and (f) mapping [from] mathematics to meaning. Figure 1 depicts Tuminaro's schematics for recursive plug-andchug.

In recursive plug-and-chug, students plug numbers to an equation and produce a numerical answer without meaningfully understanding the equation. Students playing this game generally do not exhibit conceptual understanding of the situation. In this game, students generally execute the following steps:

(1) Identify a target quantity (e.g., $\Delta E$ ).

(2) Find an equation relating the target quantity to other quantities, e.g., $E=\frac{1}{4 \pi \epsilon_{0}} \frac{q}{R^{3}} r$.

(3) Determine what quantities are unknown and substitute the values of the known quantities, e.g., $E_{1}=\frac{1}{4 \pi \epsilon_{0}} \frac{q}{(1)^{3}}(0.2)$.

(4) If one or more quantities, called subtargets, in the equation are unknown, look for additional equation(s) to find the unknowns, e.g., assume $\frac{q}{4 \pi \epsilon_{0}}=c$, a place holder for the unknowns.

(5) Once one or more subtargets have been figured out, return back to their main equation and plug in the values to get a final answer, e.g., $\Delta E=c(0.6)$.

Students pursuing the recursive plug-and-chug game often possess incomplete and/or inaccurate understanding of the mathematical aspects underlying the problem. Students playing this game exhibit what Vinner refers to as pseudoconceptual behavior [21]. According to Vinner, a student is said to have exhibited conceptual behavior if his or her response to a question involves conceptual understanding and a genuine traceable thought process. However, if the student's response to a question does not 
involve any thought process, but is basically a superficial response containing only memorized information, the behavior is said to be pseudoconceptual. Although this type of behavior may look like conceptual behavior on the surface, it does not carry any of the traits of conceptual behavior. Often pseudoconceptual behavior is the result of students' difficulties with communication in the discipline, where they use minimal effort to respond in the hopes of satisfying the teacher $[21,22]$. Pseudoconceptual behavior makes it difficult for both teachers and researchers to assess students'true understanding. Often it is a challenging task for researchers to identify and analyze participants' pseudoconceptual behavior in their research, particularly in written responses, because it is often difficult to analyze whether or not the responses involve genuine conceptual thinking.

Gire et al. defined an e-game for describing the way students analyze graphs during problem solving [23]. In this game, labeled the graphical analysis game, the target form is one or more graphs. The knowledge base may include reasoning, lexical or symbolic, formal computational, and conceptual resources. The entry condition is the presence of information in a graph or the requirement to produce a graph. Graphical analysis may include several possible moves such as interpreting lexical information (legend, axes, titles, units), creating a story, readout values, comparing data sets, identifying features, extrapolating or interpolating, making an estimation, calculating slope, calculating area, and translating to a new representation.

This framework was also used recently at a larger grain size of problem solving in physics. Chen et al. described and distinguished the two ways students partake in "answer-making" instead of sense-making or problemsolving behaviors when working on physics problems, and defined the answer making e-game to show this [24]. They argue that this e-game has two alternate paths depending on whether students' intuition or memory provides a satisfactory answer for the posed question. In one case, either memory or intuition serves the students, and they provide an answer and then justify that answer, with the justification step or move consisting of recursive sets of "conceptual reasoning" and "do math" moves. In the other case, neither intuition nor memory suffice, and students start with conceptual reasoning and do math to arrive at an answer. The primary finding here is to formalize the large-grain-sized strategies of answer-then-justify or reason-then-answer that form the two major routes to answering questions. Chen et al. point out that answer making is "like a super game" [24] [p.12] that leads to the use of other, existing e-games.

\section{Epistemological framing}

A student may possess a completely different mind set in a physics classroom as compared to a mathematics classroom. Similarly, a student may perceive two similar stimuli, with the same theme but varied contexts, such as the
FTC-based problems in the contexts of temperature and electrostatics, quite differently. Not only might a student exhibit varied attitudes from one classroom to another or from one context to another, but two students may perceive a single stimulus quite differently. These types of diverse student reactions to a single situation have been explained by Hammer and colleagues using the term framing [25]. According to Hutchison and Hammer [26], framing is one's generalization of knowledge from one's former experiences to interpret and make sense of the phenomena in the situations one considers to be equivalent. According to Hammer et al., students'framing of a situation may have various aspects, such as social, affective, and epistemological. This study focuses only on the epistemological aspect of framing. The term epistemology pertains to the question, "How do we come to know what we know?" [25].

In a study of epistemological framing that investigated student application of mathematical knowledge and skills in physics problem solving, Tuminaro found that students' strategies for solving physics problems are often determined by their initial framing of the problem [27]. He showed three types of student framings that students used while solving algebra-based physics problems: (a) rote equation chasing, (b) qualitative sense-making, and (c) quantitative sense-making. Similarly, Bing and Redish [11] described four types of epistemological framings that students exhibited while dealing with the mathematical aspects of upper-division physics problems: (a) calculation, (b) physical mapping, (c) invoking authority, and (d) math consistency. Bing and Redish claim that a student's epistemological framing may shift during problem solving, sometimes on a moment-to-moment basis. They also argue for two signs of expertise in framing: first, the dynamical blending of the 4 major framings they identified in the course of solving a problem; and second, a "more expansive framing that allows students to access and interact [with] the resources" they describe [[11], p. 9]. Thus, an epistemic game that captures expert problem solving may not be tied to a single frame, but may involve multiple frame shifts.

\section{METHODS}

We conducted semistructured individual interviews with 14 undergraduate students at a Northeast public research university, all of whom had completed at least two semesters of calculus. All the participants were enrolled in second semester calculus-based introductory physics; seven were also enrolled in multivariable calculus. The students were recruited on a voluntary basis. We collected course grades for the introductory calculus and calculusbased physics sequences for the interview participants; the range of subjects' grades in these courses indicates that the subjects can be considered representative of the student population of these courses. Of the 14 interview participants, five were female and nine were male students. Each 
interview lasted for about $1 \mathrm{~h}(15 \mathrm{~min})$. All interview sessions were videotaped using a digital camcorder.

\section{A. Interview tasks}

Four analogous problems with similar mathematical theme but different physics contexts were asked during the interviews. The solution of the problems required the application of single variable integral concepts, particularly the definite integral, which the participants were already familiar with. Despite the varied physics contexts, these questions share a common underlying mathematical theme based on the fundamental theorem of calculus: finding the change in a quantity (e.g., $\Delta x$ ) from the graph of its rate (e.g., $v$ versus $t$ ). The purpose of the physics contexts used in the problems were to mask the common mathematical theme; thus the problems could be solved by using and applying calculus concepts and skills without any physics knowledge. Although not demanded by the questions, if participants wanted to make sense of the physics contexts, they could do that using either their formal physics knowledge (e.g., velocity is the rate of change of displacement) or their real-life experience (e.g., a car moves faster when it speeds up).

Several short and situational (depending upon the student response) follow-up questions were asked following the participants' responses to each of the principal questions. Although the follow-up questions were based mostly on the participants' responses, some were designed to probe their understanding of the specific elements of the FTC including the concept of a Riemann sum, the meaning of the differential $d x$, and the relationship between a rate and its integral. Student understanding of these specific concepts was examined by analyzing the specific (relevant) moments in the video data. The results from all the four problems were consistent with each other. So we focus on only two problems in this paper that we referred to as the temperature and the electrostatics problems.

The temperature problem (Fig. 2) involved the graph of rate of change of temperature $(d T / d t)$ with respect to time $(t)$; students were asked to find the change in temperature between two specific times from the given graph. Although students usually do not deal with any temperature related context in introductory calculus-based physics, the solution to this problem did not require any formal physics reasoning. One can solve the problem simply by recognizing that the required temperature difference $(\Delta T)$ is given by the integral $\int_{t_{1}}^{t_{2}} \frac{d T}{d t} d t$.

The electrostatics problem (Fig. 3) asked to find the potential difference $(\Delta V)$, given the $E(r)$ graph and the definition of electric field as $E=-\frac{d V}{d r}$. Study participants had recently dealt with electrostatics in their introductory physics class. Because the participants were enrolled in introductory physics, the electrostatic problem was asked in a one-dimensional context involving spherical symmetry. Because of this, we did not emphasize the vector signs in
The graph below shows the rate of change of temperature with respect to time (i.e., $d T / d t)$ versus time $(t)$.

a. Between time $t=0$ and $t=1 \mathrm{hr}$, how much has the temperature changed?

b. Between $t=1 \mathrm{hr}$ and $t=2 \mathrm{hr}$, how much has the temperature changed?

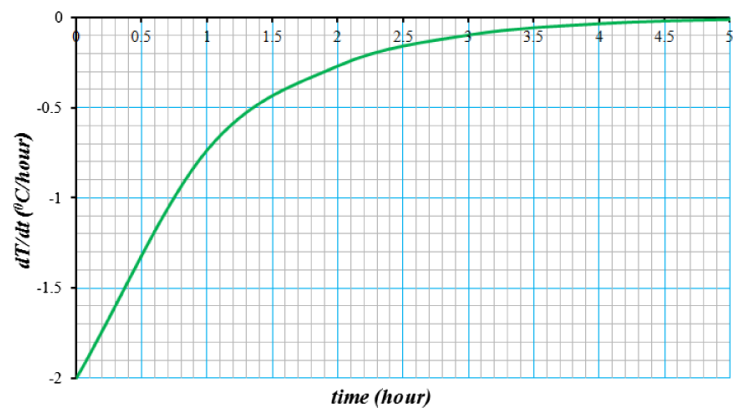

FIG. 2. The interview problem on temperature.

the problem and student solutions. In order to evaluate the potential difference $(\Delta V)$, students were expected to find the connections between $\Delta V, E=-\frac{d V}{d r}$, and the $E(r)$ graph, in particular the area under the curve. Because our focus was mostly on student application of the FTC within the graphical representation, we introduced only a thin layer of physics by using the electrostatics context. Given $E=-\frac{d V}{d r}$, one can evaluate $\Delta V$ either analytically using $\Delta V=-\int_{r_{1}}^{r_{2}} E d r$ or graphically recognizing $\int_{r_{1}}^{r_{2}} E d r$ as the area under the $E(r)$ curve without understanding the actual physics context. The required potential difference $\Delta V$ in the electrostatics problem could be evaluated analytically using the expression for electric field. Although the expression for the electric field $\left[E(r)=\frac{1}{4 \pi \epsilon_{0}} \frac{q}{R^{3}} r\right]$ was provided in the problem, no information about charge and $\epsilon_{0}$ was provided. Thus, to determine a numerical answer for $\Delta V$, the area under the curve needed to be evaluated. This could be achieved by estimating the number of squares under the curve. However, we expected that this would not be obvious to the targeted students because previous studies in mathematics and physics education have shown that students have difficulties connecting integrals and their graphical representations, such as area under the curve and the Riemann sum [2,18,28,29].

\section{B. Interview analysis}

Initially, the interview data were analyzed on the basis of grounded theory [30]. We started the analysis by writing memos describing and interpreting (making sense of) the student responses in the interviews. Memos were written during and immediately after the data collection process (interviewing) to document some of the 
Electric field and electric potential are related by

$$
E(r)=-\frac{d V}{d r}
$$

where $E$ is electric field, $V$ is potential and $r$ is position of the field.

The figure below shows the magnitude of the electric field inside and outside a uniformly charged insulating sphere of radius $R$. Find the potential difference between two points

(a) $r_{1}=0.2 \mathrm{~cm}$. and $r_{2}=0.8 \mathrm{~cm}$.

(b) $r_{1}=0.6 \mathrm{~cm}$. and $r_{2}=1.6 \mathrm{~cm}$.

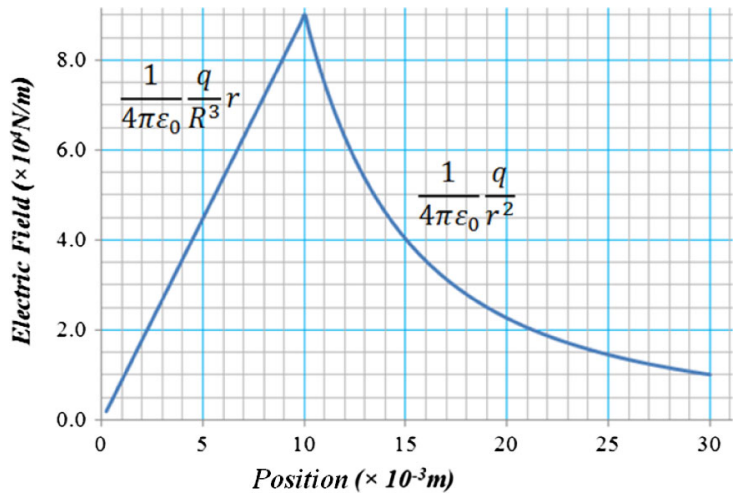

FIG. 3. The interview problem on electrostatics.

observations made during the process. The postinterview memos were written by watching and rewatching the recordings several times both individually and with another researcher. The second researcher confirmed the features and patterns in student responses identified by the first researcher. The individual interviews were turned into textual material, which became the primary data for subsequent analysis. The transcription of the audio-visual data was carried out in a commercially available software called Inqscribe ${ }^{\circledR}$. The audio visuals were watched several times to assure the correctness of the transcripts. Because of the technical nature of the research, the transcripts were not created completely verbatim. Technical terms (e.g., delta, integral, plus) were presented in the conventional form (e.g. $\Delta, \int,+$ ) to make the interview transcripts more readable. In addition, nonverbal communication such as gesture, laughter, or sighing was not included in the transcript.

Using grounded theory-based analysis, we found that students used three types of mathematical skills, separately or in combination, to solve the interview problems. These skills include using algebraic, graphical, and integration skills [19]. However, this classification of student strategies did not inform us much about students' epistemological stance while solving these mathematically based physics problems, specifically problems so centered on graphical representations. Thus, we used the analytical framework of epistemological framing to investigate student epistemology while dealing with FTCbased physics problem solving. Furthermore, we found all four components that constitute an epistemic game in almost all the student problem-solving strategies including knowledge base, epistemic forms, starting and ending conditions, and moves. This motivated us to use epistemic games as a guiding framework for analyzing the data. Nevertheless, our research methods (e.g., clinical interviews) also satisfy the requirement for using both of these perspectives as analytical frameworks; the frameworks chosen best fit our data because of the nature of analysis, such as an individual's application of knowledge resources in problem solving. As mentioned above, these frameworks have been used by previous researchers in similar studies, i.e., student application of mathematics in physics problem solving.

In order to identify and categorize the epistemic games played by students during problem solving, we focused on the following five components in individual problemsolving strategies:

- Epistemic form-The target structure that guides the inquiry, e.g., equations, graphs, pictures, a flow chart.

- Knowledge base-Knowledge elements and/or skills used by students during problem solving, such as simple algebraic skills, graphical reasoning, or integral concepts.

- Entry and exit conditions-Specific element(s) in a problem that motivate students to choose a particular strategy, such as rate and/or integral equations, presence of graph, specific physics terms.

- Moves-Steps students make to fill the epistemic forms, such as equation rearranging, physical or graphical interpretation, use of mathematical operations, number substitution.

- Constraints-Either explicit or implicit conditions imposed by the problem or a problem solver, such as linearity of the function $\left(E=\frac{1}{4 \pi \epsilon_{0}} \frac{q}{R^{3}} r\right)$. When a constraint is not explicitly indicated in the problem, the problem solver may impose one by making certain assumptions or approximations, such as constancy of the integrand $E(r)$.

Our primary goal for using the perspective of epistemic games was to identify specific patterns and components of student strategies for solving mathematically based physics problems. With some modification of the relevant games, we were able to label most-but not all—of the observed student strategies as existing games. In particular, the structures of epistemic games proposed by Collins and Ferguson [20], Tuminaro and Redish [9], and Gire et al. [23] matched very closely with the structures of some of the strategies manifested in this study. One particular student strategy that was commonly manifested by students while dealing with mathematically based physics problems did not fit into any of the previous e-games. Thus we propose 
a new e-game, the analytical derivation game, to describe this strategy.

\section{RESULTS}

Our analysis based on grounded theory revealed that the majority of the students in this study solved both the temperature and the electrostatics problems analytically by rearranging the given equations and then performing integration on both sides of the equations. Using the epistemic games perspective, we refer to this analytical problem-solving mode as the analytical derivation game. Only a few students identified that the problem could alternatively be solved by evaluating the area under the curve, and even these students initially chose to play the analytical derivation game because they considered that this game would yield a more accurate result. Below we present examples of various ways students play this game.

\section{A. The analytical derivation epistemic game}

A detailed analysis of this strategy, using the lens of epistemic games, indicates the presence of all the essential components that characterize an epistemic game (see Fig. 4). The epistemic form for this game is a series of equations. Playing this game involves establishing the relationships between different variables for physical quantities in hope to evaluate the required physical quantity. The knowledge base students may use in this game are procedural, symbolic, computational, and, less often, conceptual resources. The entry condition is the presence of an equation or an algebraic expression or the requirement to find an expression, whereas constraints are explicit or implicit conditions imposed by the problem or the problem solver. Examples of constraints are assuming the constancy of the function $\frac{d T}{d t}$ and approximating the curve as a linear or a quadratic function. There are numerous ways in which a student may make moves while playing this game, such as cross multiplying, substituting, integrating, approximating an integrand as an algebraic expression, and making assumptions about integrands. Usually students do not make sense of their final expression in this game, neither mathematically nor physically. The exit conditions could be the derivation of an acceptable equation, evaluation of a numerical answer by substituting numbers in the final equation, obstruction from not finding any further way, or dissatisfaction with one's own intermediate steps or final result. These characterizations of the components of this epistemic game are based on our analysis of interview data through the epistemic games perspective. Our claim is that the four components mentioned above are unique to this strategy, making it a distinct game.

Although the students in Tuminaro and Redish's study manifested some degree of symbol manipulation moves (e.g., in the recursive plug-and-chug game), they do not constitute an analytical derivation game because the

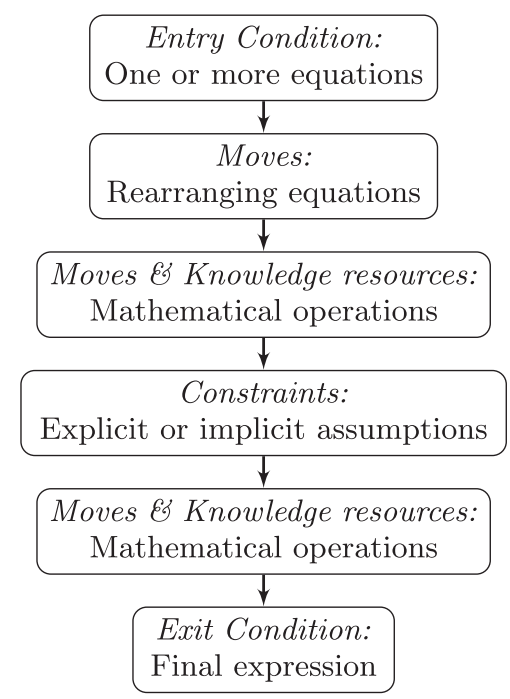

FIG. 4. Components of the analytical derivation epistemic game.

expressions students use in recursive plug-and-chug are formulas that are memorized straight from textbooks (e.g., $P V=n R T)$. In analytical derivation, students start with one or more expressions that may or may not be textbookbased formulas. The majority of a student's focus revolves around one or more raw starting equation(s). While playing this game, a student performs different kinds of mathematical operations, such as cross-multiplication, integration, distribution, differentiation, etc., on the raw equation(s). Some students may substitute numbers during intermediate moves, some may hold off substitution until the final expression, while others may not use numbers at all, neither in intermediate moves nor in the finished expression or equation. In contrast, during the recursive plug-and-chug game, students constantly focus on the numerical answer.

Our analysis of student problem-solving strategies in this study, using the perspective of epistemological framing, revealed that students were in one or the other frames proposed by Tuminaro and Bing and Redish. Similarly, using the perspective of epistemic games, we found that students pursued different e-games proposed by previous researchers for solving the mathematically based physics problems. The next sections document a number of student strategies for solving the temperature and the electrostatics problems to manifest the distinctions between the recursive plug-and-chug game and the analytical derivation game. We explore the implications and the potential pitfalls of these games and show how some students use these games to move towards the right solution paths.

\section{B. Epistemic games for the temperature problem}

Although most of the students attempted the temperature problem using analytical derivation, only three students 
A student response

E-game components
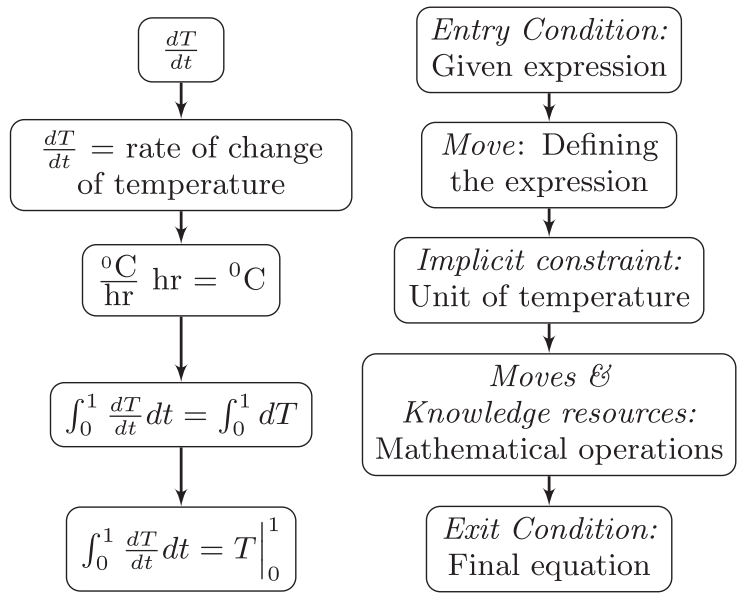

FIG. 5. Example of the analytical derivation epistemic game for solving the temperature problem.

correctly derived the target form-the expression for $\Delta T$-and only two of these connected the resultant equation with the given graph to find the desired quantity. Students manifested various kinds of moves to derive the target form.

A diagram of the general version of analytical derivation applied to the electrostatics problem is shown in Fig. 5. Students who pursued analytical derivation integrated the rate term $\left(\frac{d T}{d t}\right)$ with respect to time to derive the change in temperature (i.e., $\int_{0}^{1} \frac{d T}{d t} d t$ ). Because there were no analytical expressions given for the rate of change temperature as a function of time in this problem, some students approximated the given curve with an algebraic function (e.g., linear), where the others considered the temperature itself to be an algebraic function. But only a few students were able to connect the target form, i.e., the definite integral expression, with the area graph and hence the area under the curve. However, once they realized that they needed to find the area under the curve, they considered either counting the number of squares under the curve and multiplying it by the value of a unit square or forming simple geometric shapes, such as right triangles and/or squares, to find the required quantity.

We present three cases to exhibit the different ways that students solved the temperature problem using the analytical derivation game. But first we start with a case of recursive plug-and-chug to demonstrate the differences between the two games.

\section{Case A1: Alex playing recursive plug-and-chug}

The interviewer explained the temperature problem in detail at the onset of its administration. Because the temperature problem did not include an equation, Alex

focused primarily on the graph. After spending a few seconds on the graph, Alex responded.

1 Alex: So, umm, basically my thought for this one
2

3

4

5

6

7

8

9

10

11

12

13

14

\begin{abstract}
Alex: So, umm, basically my thought for this one ture over time and we need to find the change in temperature between time of zero [hour] and time of one [hour], we look at the start value of $[d T / d t]$, time at zero and we see that's -2 there. We get the start, umm, umm, sorry the finish of where is at the time at one hour $[d T / d t(t=1 \mathrm{hr})]$. Umm, you know, it looks like it's about -.75 ish $\left[{ }^{0} \mathrm{C} / \mathrm{hr}\right]$. So there is a change of about 1.25 , umm, positive umm... So my best guess would be a positive change of 1.25 ish, umm, degrees per hour at that point.
\end{abstract}

Interviewer: No, the question is not rate... Alex: Oh, umm, 1.25 degree Celsius per... I: I want temperature change. How did you calculate that?

Alex: I looked at the starting value, so we started at time zero. So we are starting at this start point and then our end point is at time equals 1 [hour] and so it's basically, it's kind of finding the slope in a way. So you have the two $y$ values and then you have the $x$ values. So you have $y_{2}-y_{1}$ and $x_{2}-x_{1}$ on the bottom $\left[\frac{y_{2}-y_{1}}{x_{2}-x_{1}}\right]$ and so...

I: Why are you finding slope here? What do you get by finding slope?

Alex: Umm, by finding slope, you come out with negative... I'm gonna write... We have -.75. I'm just gonna say that's just about -.75 for the start, ah for the end there, minus negative 2, 'cause that's the beginning value. Umm, that's the, umm, $y$ point value and then we have a finish at $x=1$ and the start of zero so that's $1-0$ and so we come out with about 1.25 , umm, for the change in temperature [referring to $\frac{-0.75-(-2)}{1-0}=1.25$ ]. I: So this is... what...

Alex: That's what I'd say the best I know is of what change in temperature would be, just by judging it.

I: So you are just finding slope to find change in temperature?

Alex: Umm, pretty much, umm, I am just looking at like how much is changed, umm, at the start versus at the finish is basically the slope.

In this example, Alex's goal was to find a numerical value for the change in temperature $(\Delta T)$. His target form was the slope equation. He played the recursive plug-and-chug game by first determining and then plugging the values of $d T / d t$ corresponding to $t_{1}$ and $t_{2}$ in the generic slope equation $\frac{y_{2}-y_{1}}{x_{2}-x_{1}}$, where $y$ and $x$ represent $d T / d t$ and $t$, respectively. In this game, he did not use any mathematical operation to derive the expression for the change in temperature $(\Delta T)$. He simply used the slope formula to determine the corresponding numerical values. 


\section{Case A2: Kara's unit-based analytical derivation game}

In response to the temperature problem, Kara immediately suggested that she needed to somehow evaluate the integral of the rate of change of temperature over the given time interval to find the change in temperature. She analyzed the units of the integrand and the differential to assure that her moves were leading to the right path. As she solved the problem, she explained.

1 Kara: So if I integrate $d T / d t$ [writes $\left.\int \frac{d T}{d t}\right] \ldots$

2 So this [referring to $\frac{d T}{d t}$ ] is rate of change of

3 temperature per time. So if I integrate that

4 you get a rate [sic, temperature]. Then I

5 should get just temperature [writes $\int \frac{d T}{d t} d t=$

$6 \int$ rate of change of temp per time $\rightarrow$ Temp]. So I

7 think, maybe, I just have to integrate that. Okay,

8 that makes more sense. So I have integral of...

9 umm, $d T / d t$ times $d t$. So that's really degree Cel-

10 sius per hour [times] hour, so my hours cancel out [refer-

11 ring to $\frac{{ }^{\circ} \mathrm{C}}{\mathrm{hr}} \mathrm{hr}$ ]. Then if I integrate over it, it will

12 give me just the temperature, hopefully [writing

$\left.13 \int \frac{{ }^{0} \mathrm{C}}{\mathrm{hr}} \mathrm{hr}=\mathrm{Temp}\right]$.

14 I: Okay.

15 Kara: So, now I have to do it... So from time

160 to time $1 d t$ [writes $\int_{0}^{1} \frac{d T}{d t} d t$ ], that will give me,

17 that's really just the integral from 0 to $1 d t$ equals

$18 T$ [writes $\int_{0}^{1} \frac{d T}{d t} d t=$

$\left.\int_{0}^{1} d T=\left.T\right|_{0} ^{1}\right]$. I don't know

19 I am screwing that up. No... Okay, is this, is

20 this the temperature, change in temperature over

21 time?

22 I: Right, um hum.

23 Kara: So I think I actually have to go from...

24 I: That's the rate.

25 Kara: Okay, I think I want to go from ... Sorry I

26 am thinking...

27 I: No that's fine, perfectly fine.

28 Kara: I wish I could think of Calc 1 right now.

29 So ....ok So I have the rate of change of tempera-

30 ture over time... [mumbling] It should just give me

31 temperature. I feel like I have to multiply some-

32 thing and I am not sure what. Okay... $d T / d t$ and

33 they go from ... Yup I am stuck again, yeah.

As seen in the above excerpt and in Fig. 6, Kara attempted to solve the problem analytically without realizing that there was no analytical function to be integrated. Her strategies in lines 1-9 satisfy well with the characteristics of the analytical derivation game. Her epistemic form in this game was a series of equations. She pursued the mathematical procedure for the integration to complete her epistemic form. In lines 5-6, she derived an analytical expression for the temperature and used the units of $\frac{d T}{d t}$ and $d T$ to verify the validity of her equation. But she did not seem satisfied with her final expression and, after analyzing her moves, finally gave up when she was unable to find

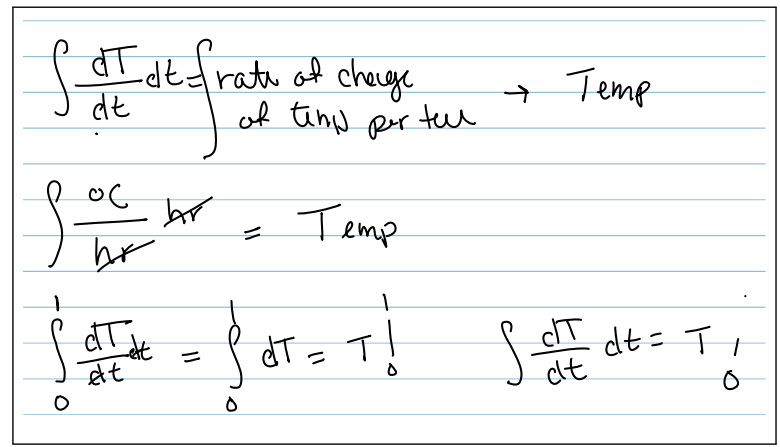

FIG. 6. Kara's analytical derivation game to solve the temperature problem.

the connection between the definite integral that she constructed and the given graph.

\section{Case A3: Jake playing analytical derivation by approximating an algebraic function}

This case exemplifies how some students prefer integrating an algebraic function that approximates the curve rather than using the graph to approximate the area under the curve. Nguyen and Rebello [2] also identify this tendency, noting that students used the graph to find the integral only after ruling out the analytical solution, either for its complexity or for lack of a function. In this case, Jake first looked at the temperature problem quietly for a moment and wrote down the equation $\Delta T=\frac{d T}{d t} \Delta t$. When the interviewer asked him to explain the equation, he responded.

1

2

3

4

5

6

7

8

9

10

11

12

13

14

15

16

17

18

19

20

21

22

23

24

25
Jake: So, if the temper..., the change in temperature is equal to the change in temperature over time to the change in time [referring to $\left.\Delta T=\frac{d T}{d t} \Delta t\right]$. So, it should be an equation, I think, should be similar to this, umm, so... I: How do you know that equation?

Jake: Umm, this is a... I think this is a form of math linear approximation equation, I think, that's from math and I think I can use this to solve the problem.

I: Okay.

I: So, how do you get $\Delta T$ then?

Jake: How do I get $\Delta T$ ? Umm... [after a long pause] Sorry, I'm trying to...

I: It's okay.

Jake: So you can take the, I know you can take the integral under the curve to find the total change. Sorry, it's just been a long day.

I: It's okay, you can take time.

Jake: Okay. [A long pause while looking at the problem]

After the long pause, Jake proceeded by approximating the given curve with the function $\ln (x)-2$ and writes its integral as $\int_{0}^{1} \ln (x)-2$ (without $d x$ ), then he explained: 
26 Jake: Umm, so, it's kinda hard to, like, figure

27

28

29

30

31

32

33

34

35

36

37

38

39

40

41

42

43

44

45

46

47

48

49

50

51

52

53

54

55

56

57

58

59

60

61

62

63

64

65

66

67

68

In the above excerpt, Jake proposed two approaches to deal with the problem. Initially, he derived the expression for $\Delta T$ by making a "linear approximation" of the curve (a constraint) and using the units of the vertical and horizontal axes. He then switched the constraint by approximating the curve as the function $\ln (x)-2$ and realized the need for performing an integration to solve the problem. In both approaches, he considered that the solution to the problem required either deriving an equation or routine mathematical operation (integration). For the latter approach, Jake approximated the given curve with the function $\ln (x)-2$ and wrote its integral as $\int_{0}^{1} \ln (x)-2$ (without $d x$ ). Jake realized that the required quantity could be evaluated by finding the area under the curve, and even suggested that this could be done by counting the squares. However, he

was reluctant to use that approach because he thought that it would be a lengthy process. Although his approximated curve was not necessarily any more accurate than estimating some of the irregular squares to find the area under the curve, he still preferred the analytical approach over the graphical.

A few other students also expressed their preference for an analytical approach over counting the squares to evaluate the required quantity due to a concern with the accuracy of the process. They considered that the analytical approach would result in a more accurate answer even when the exact algebraic function (integrand) was not provided.

\section{Case A4: Andrew beyond the analytical derivation game-connecting integral and area under the curve}

In response to the temperature problem, Andrew first wrote $\int_{0}^{1} d T$, then he erased $d T$ to replace it by its rate, i.e., $\int_{0}^{1} \frac{d T}{d t}$. After a while, he added the missing term in the integral $(d t)$ to make the integral $\int_{0}^{1} \frac{d T}{d t} d t$. The interviewer then asked him how he would find a numerical answer for the change in temperature.

1

2

3

4

5

6

7

In order to elicit Andrew's understanding of the connection between the integral and the area under the curve, the interviewer asked him why he thought the integral that he wrote was exactly what was being asked in the problem and how the area under the curve would give the desired quantity. His response was as follows:

8 Andrew: $\frac{d T}{d t}$ represents this curve [pointing to

9 the graph], you integrated over time to get your

10 change in temperature.

11 I: Okay. So what does this [indicating the integral] 12 represent in the graph?

13 Andrew: This part right here [indicating the area

14 under the curve between 0 and 1 hour time].

15 I: Can you mark that with the pen?

In response, he drew a boundary, encompassing the space under the curve between 0 and 1 hour time, on the graph to show the area that represented the integral of $\frac{d T}{d t}$. Throughout the problem-solving process, Andrew did not invoke any physical sense making. He first played the analytical derivation game, where the epistemic form was a series of equations, which he completed by making the moves described above. Then with the interviewer's prompt, he pursued the graphical analysis game in order to find the connection between the integral and the graph, in which the epistemic form was the given graph and the 


\section{A student response E-game components}

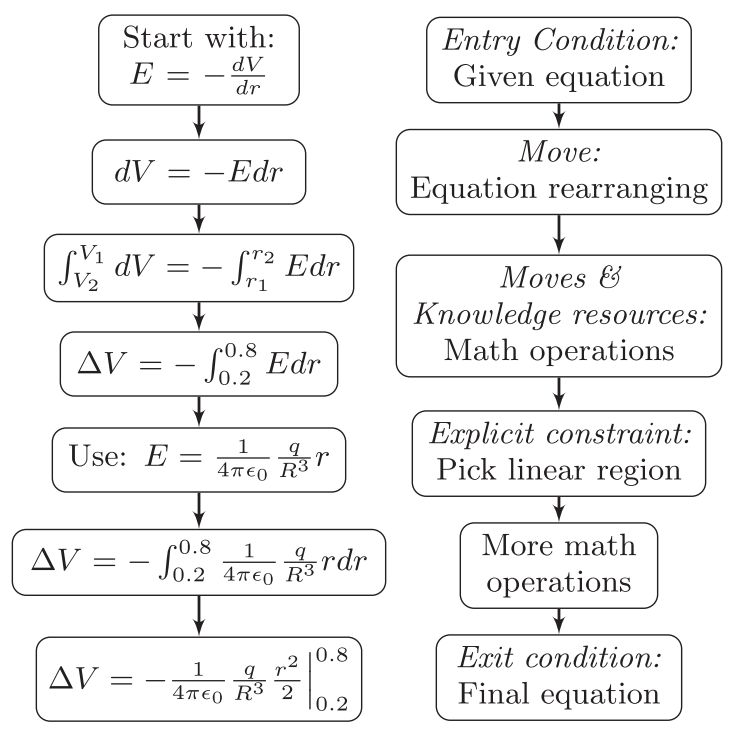

FIG. 7. Example of the analytical derivation epistemic game for solving the electrostatics problem.

moves were to analyze it to recognize the relevant graphical feature, which was the area under the curve. This case exemplifies how a few students proceeded beyond the analytical derivation game and successfully associated it with a secondary game, the graphical analysis game, by recognizing the graphical representation of integral as the area under the curve.

\section{Epistemic games for the electrostatics problem}

A diagram of the general version of analytical derivation applied to the electrostatics problem is shown in Fig. 7. Most of the participants started the problem by rearranging the given rate equation $E=-\frac{d V}{d r}$ to $d V=-E d r$. Then, to evaluate the potential difference inside the sphere, they replaced $E(r)$ with $E(r)=\frac{1}{4 \pi \epsilon_{0}} \frac{q}{R^{3}} r$ and solved the integral analytically. Since there was no information provided about the charge and the value of $\epsilon_{0}$, students simply left their final answers in notational form, e.g., $\Delta V=\left.\frac{q}{4 \pi \epsilon_{0}(1)^{2}} \frac{r^{2}}{2}\right|_{0.2} ^{0.8}$. Although the majority of students identified the essence of integration for transforming the differential $(d V)$ to the difference $(\Delta V)$, most of them did not connect their integral to the relevant graphical feature, namely, the area under the curve. Only when the students were asked for an alternative way to find the required quantity did they state that it could be found by evaluating the area under the curve in the given graph. However, as with the temperature problem, the majority of the students did not feel comfortable using the graph because they needed to approximate the number of squares to find the area under the curve and they considered that this would not give an exact answer. They considered analytical derivation as the most appropriate game because

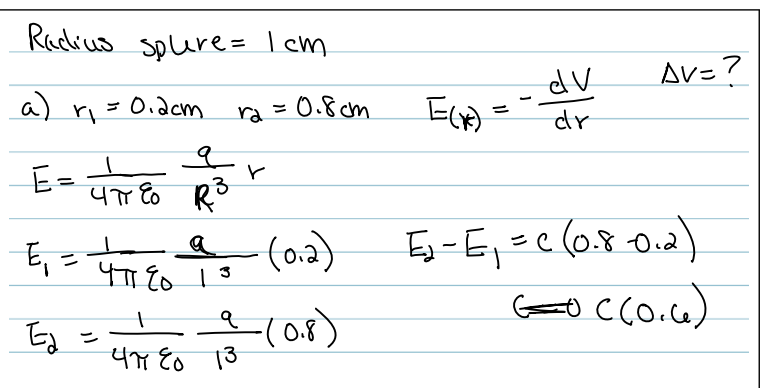

FIG. 8. Kara's initial, recursive plug-and-chug solution to the electrostatics problem.

they could get the exact answer only through such a game. We present four representative cases to exhibit different ways students played analytical derivation while solving the electrostatics problem.

\section{Case B1a: Kara's recursive plug-and-chug game}

While most of the students rewrote the given expression $E(r)=-\frac{d V}{d r}$, then rearranged the equation in terms of $d V$, some students simply inserted the given values in the expression for the electric field without clearly envisioning how they would using that expression to find $\Delta V$. As shown in Fig. 8, Kara first started the electrostatics problem by rewriting the given numerical quantities for $r_{1}$ and $r_{2}$ and the given expressions $\left[E(r)=-\frac{d V}{d r}\right.$ and $\left.E=\frac{1}{4 \pi \epsilon_{0}} \frac{q}{R^{3}} r\right]$. After substituting the known numerical values into the expression for $E$, she arrives at the final expression $E_{2}-E_{1}=c(0.6)$, in terms of the unknown constant $c$.

Although Kara's initial strategy might superficially be thought of as an analytical derivation game, a more detailed examination revealed that it was indeed recursive plug-and-chug. In order for a strategy to qualify as an analytical derivation game, one needs to derive an equation for the quantity being asked through a series of steps using various resources, such as integration and graphical features. However, her initial target form was "finding a difference," for which she targeted the quantity $E_{2}-E_{1}$; she simply plugged in the values of the known quantities in the given expression for $E$. Then she denoted all the unknown constants as a letter $c$ and expressed the potential difference as $E_{2}-E_{1}=0.6 c$ without exhibiting the use of any sophisticated mathematical resources such as integration. She did not clearly envision, when she began the

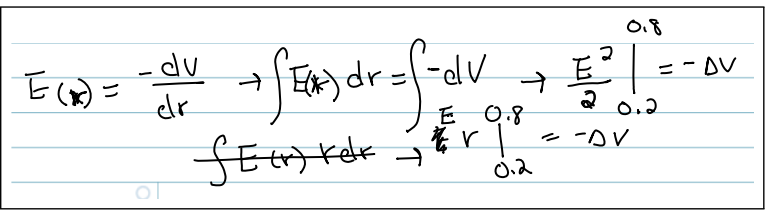

FIG. 9. Kara's version of the analytical derivation game to solve the electrostatics problem. 
solution, how she would use the result, i.e., $E_{2}-E_{1}$ to find $\Delta V$. Her rote mode of problem solving, in which she mindlessly plugged in numbers in given equations, was more consistent with the characteristics of the recursive plug-and-chug game.

\section{Case B1b: Kara switching to the analytical derivation game}

When asked why she was evaluating $E_{2}-E_{1}$, Kara responded that she did not know why she was doing that. Then she approached the problem in a completely different way as shown in Fig. 9.

After completing her solution, she explained.

$\begin{array}{ll}1 & \text { Kara: So, I think... So, I'm looking for a delta } \\ 2 & V \text { [pointing at } \Delta V \text { ] really. So if I multiply my } \\ 3 & d r \text { over... That's an } r \text {, oops, then I have the in- } \\ 4 & \text { tegral of... oh, and then I integrate... that's the } \\ 5 & \text { integral of } E(r) d r \text { equals the negative the integral } \\ 6 & \text { of } d V \text { [pointing to } \int E(r) d r=\int-d V \text { ]. So then if } \\ 7 & \text { I integrate this [referring to } \int E(r) d r \text { ], I get } E^{2} / 2 . \\ 8 & \text { Umm, and I'm going from... it's } d r \text { [points at } d r \\ 9 & \text { in } \int E(r) d r \text { ], so I take my } r \text { values, which is } r_{1} \\ 10 & \text { is } 0.2 \text { cm to }\left[r_{2}=\right] 0.8 \text { cm [pointing at the limits } \\ 11 & \text { in }\left.\frac{E^{2}}{2}\right|_{0.2} ^{0.8} \text { ]. Umm, that'll give me negative delta } V \\ 12 & \text { [pointing at }-\Delta V \text { ]. So if I just plug... okay, well } \\ 13 & \text { I'll have to plug... here's my } 0.2 \text { into here [refer- } \\ 14 & \text { ring to } E_{1}=\frac{1}{4 \pi \epsilon_{0}} \frac{q}{1^{3}}(0.2) \text { in her previous strategy] } \\ 15 & \text { and that'll give me } E .\end{array}$

In the above excerpt, Kara was treating the integrand $E(r)$ as the variable of integration (i.e., integrating with respect to $E$ instead of $r$ ), which was her self-imposed constraint. At this point the interviewer momentarily intervened and reminded Kara that the integrand $E$ was a function of $r$ and she could not just integrate it without expressing it in its functional form first. After the interviewer's intervention, she then wrote $\int E(r) r d r \rightarrow$ $\left.E r\right|_{0.2} ^{0.8}=-\Delta V$ and after drawing a striking line over $\int E(r) r d r$ (see Fig. 9), she responded.

16 Kara: So... now, if I take the difference in my $r \mathrm{~s}$, 17 which is 0.6 [pointing over $\left.\mid \begin{array}{l}0.8 \\ 0.2\end{array}\right] \ldots$ or is it negative

18 0.6? And then I plug it into the... See I needed

19 to find $E$ [pointing at $E=\frac{1}{4 \pi \epsilon_{0}} \frac{q}{R^{3}} r$ ], but now

20 it's the integral of $E$ [pointing at $\int E(r) d r$ ]... and

21 the electric field isn't constant, so I cannot pull it

22 outside the integral.

23 I: [Pointing at $\left.E r\right|_{0.2} ^{0.8}$ ] So what you, what you did 24 there?

25 Kara: Well, I tried..., ah...

26 I: This one [pointing at $E(r)$ ], what's this?

27 Kara: I took the integral of $E d r$.

28 I: So you are... you are considering $E$ as constant?

29 Kara: Right, but I can't do that.

30 I: Right it's not constant.

31 Kara: Right. So I need to... E isn't constant,

32 when I integrate it. Will it still be $E^{2} / 2$ then?

33

34

35

36

37

38

39

40

41

42

43

No. So if I integrate $x$, I'll get $x^{2} / 2$. No, I won't, that's not true. Integrate $x^{2} \ldots$ Wait a minute.

Okay, so $E$ isn't constant, but I am integrating with respect to $r$, so I can't just keep my $E$ there. Like, would $E$ go to zero then? I don't know. Well, yeah 'cause it's not with respect to $r$, so $E$ goes away, right [scratches $E$ in $\left.\left.E r\right|_{0.2} ^{0.8}\right]$ ?

I: How $E$ goes away?

Kara: Oh, now I am confused. That's... No, it can't go away because that doesn't make sense ... [rewrites $E]$.

Because Kara seemed to be confused with the outcome of her strategy due to her activity in the analytical mode, the interviewer asked her if there was any other way that she could solve the problem. She responded that she could simply count the squares to find the required quantity. When the interviewer asked how the area under the curve would give the required answer, she responded that she simply remembered that from her calculus class that when it involved an integral, she often evaluated the area under the curve to determine the quantity being asked for. In order to invoke her understanding of the connection between the integral and its graphical representation, the interviewer again asked her how the area under the curve would give $\Delta V$. However, she simply elaborated her analytical solution without saying much about its connection with the graph, so the interviewer once again asked her about an alternative.

44 I: How else can you do that?

45

46

47

48

49

50

51

52

53

54

55

56

57

58

59

60

61

62

63

64

65

66

67

68

69

70

71
Kara: I mean, aside from counting the squares, which is what I don't want to do...

I: Why you don't want to count squares?

Kara: 'Cause it won't be exact.

I: I don't want exact answer...

Kara: Okay.

I: ... it's fine.

Kara: I mean, yeah I guess, what I would do is count squares, then.

I: So, my, my question is again, like, how do you connect this $\Delta V$ and counting thing, area under that line?

Kara: Umm, because...

I: How these two are connected? You are saying this is equal to that, so how?

Kara: Because my... Umm, my final is... Okay, how am I gonna explain that? I am looking at, so like, the area between two curves I guess what I would be looking at. But my first curve is zero. No that doesn't make sense. So you want me to tell you why $\Delta V$ is the area under the curve?

I: Yeah.

Kara: Oh boy.

I: It seems like you have some instinct that should, that should be equal to area under the curve, but how?

Kara: But why? Yeah, I don't know. if I can 


$\begin{array}{ll}72 & \text { explain why. Umm, so... } \\ 73 & \text { I: What exactly is the area under the curve? } \\ 74 & \text { Kara: That... area under the curve, it's like... } \\ 75 & \text { I: Like, how mathematically, in calculus... What } \\ 76 & \text { thing you use to represent the area under the } \\ 77 & \text { curve? } \\ 78 & \text { Kara: The integral. }\end{array}$

Despite possessing all the knowledge required to produce a correct solution, Kara initially followed incorrect paths to solve the problem. This may be due to her failure to identify the problem correctly as a graphically based FTC problem, preventing her from finding the right connections within her fragmented knowledge. But as the interviewer went on probing deeper and deeper-asking follow-up questions-eventually, she was able to identify that the integral could be evaluated by determining the area under the curve. However, she was unable to tell why the integral was represented by the area under the curve, suggesting that this is pseudoconceptual behavior. The set of excerpts here suggests a lack of deep functional understanding of the definite integral. This result aligns with findings by previous researchers showing students' difficulties with integrals and their graphical representations [2,18,28,29].

\section{Case B2: Joe's multiple approaches to the analytical derivation game}

In response to the electrostatics problem, Joe wrote down the integral $\int E d r$, followed immediately by $1 /\left.2 E^{2} r\right|_{A} ^{B}$ and said that that was the required potential difference. When the interviewer asked him, pointing at $\int E d r$, why he took the integral, he erased the $1 /\left.2 E^{2} r\right|_{A} ^{B}$ and responded

$\begin{array}{ll}1 & \text { Joe: Umm, it's the sum of the electric field times } \\ 2 & \text { the delta } r \text { s }[E d r] . \\ 3 & \text { I: So, what does that [the integral] give you? } \\ 4 & \text { Joe: The total potential difference. } \\ 5 & \text { I: How do you know that potential difference is } \\ 6 & \text { integral of } E d r ?\end{array}$

In response to this question, he implicitly invoked the expression for potential energy difference $\left(\Delta U=\int F d r\right)$ and recognized that $q U=V$ and $q F=E$; thus giving $q \Delta U=\int q F d r$ or $\Delta V=\int E d r$. The interviewer then asked him how he would get potential difference from the integral. His response was

7 Joe: This is the change in potential [pointing at

$8 V(B)-V(A)]$, umm [adds the limits over the in-

9 tegral] and umm this is the sum of the change

10 [pointing at the integral] that occurs if [pointing

11 at $E$ ] that's the derivative [referring to $E=-\frac{d V}{d r}$ ].

12 So that's the same thing [referring to $V(B)-V(A)$

13 and $\left.-\int_{A}^{B} E d r\right]$.

When asked how to find a numerical answer for this integral, Joe replaced the limits $A$ and $B$ with their

numerical values, .2 and .8 (in centimeters). Then after converting into meters, he replaced the numbers by .002 and .008 , respectively, and continued.

14 Joe: You gotta express $E$ in terms of $r$, which

15

16

17

18

19

20

21

22

23

24

25

26

27

28

When the interviewer asked him to show how he would find the integral, he evaluated the integral in terms of unknown constants as shown in Fig. 10. Then the interviewer asked how he would get a numerical value without knowing the values of the constant. The interviewer rephrased how else he would get a numerical answer without the values of the constants given. Looking at the graph, he immediately responded.
29

30

31

32

33

34
Joe: Ten times one-half of ten [referring to the area of the triangle formed by the straight line]. I: How?

Joe: Ah, oh, rather the, ah..., well, the area under the curve between those two points [pointing at the limits], right?
In the above example Joe initiated his solution with the analytical derivation game yielding the expression for $\Delta V$ in terms of the integral. From the resulting expression, he identified that the required quantity $(\Delta V)$ could be evaluated by evaluating the area under the curve. This example shows that students often pursue the analytical derivation game before exploring the other paths providing a bridge to explore further games, such as graphical analysis.

Although Joe eventually realized that the potential difference could be determined by evaluating the area under the curve, he was unable to tell how the area and the integral were related when the interviewer asked him how his result from the analytical derivation game was equivalent to finding the area under the curve. In addition to Joe, many other subjects, including Jake in the following example, were also unable to see the deeper connections between the integrals and the area under the curve.

\section{Case B3: Jake's preference for the analytical derivation game over using the relevant graphical feature}

In response to the electrostatics problem, Jake integrated the given function $E(r)$ between the given limits similar 


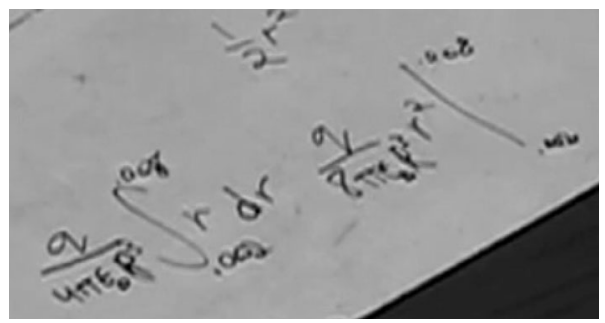

FIG. 10. Joe's version of the analytical derivation game to solve the electrostatics problem.

to how Joe responded in lines 1-4. Like Kara and Joe, Jake also identified this problem as the one requiring an analytical approach, which led him to pursue the analytical derivation game. In order to probe his perspective on an alternative strategy, the interviewer prompted him to provide an alternative approach for evaluating the required quantity.

I: That's right, I mean, you are doing integration, so, how would you, how else would you do?

Jake: Umm... I guess you could compute the ar-, you could compute the area but that doesn't tell you, like, it leaves out all the variables and it's just, it's just the area of the graph and then you

have to use the graph to figure out but I mean this is the best way to do it.

I: If you were not given this [pointing at $E=$ $\left.\frac{1}{4 \pi \epsilon_{0}} \frac{q}{R^{3}} r\right]$, how would you do that problem?

Jake: Well... like, well you can approximate the f..., you can approximate a function by looking at the slope. But you have to calculate out the slope, pick two points, figure out what the slope is for this 'cause it's a straight linear line. So, it isn't that hard for the (a) but for (b) it would be little harder cause then you have these sloping lines and you have to find an equation that will fit that. Something, ah $x^{2}$ would probably be a good function to fit that, I mean can obviously see now it's $1 / x^{2}$ but...

I: I mean without using integration, I mean, function and integration, how would you do that?

Jake: Without using analytical integration?

Umm... I guess I would just try to approximate best I could.

I: How would you estimate?

Jake: You can... you, the boxes to sort of get a rough estimate of how many there are, how much potential there is...

I: How do you know that the boxes give what you need?

Jake: Umm, if you're doing in that way, you don't unless they tell you that's what it is or you know from previous experiences.

I: I mean, how is potential difference connected to the boxes you're going to count?

Jake: Oh, because, umm, the potential difference is umm, it's a force generated by electric field over

40 the force that the electric field exhibits at distance.

41 So, you can see that, that's what it is. But it's

42 kind of hard to 'cause you don't have the equa-

43 tion for that, so you don't know what exactly the

44 ratio is for the force to distance. So, equations

45 just make everything lot easier.

When pressed for an alternate solution method, Jake immediately invoked area under the curve, but then rejected it as an approximation or estimation of the integral rather than an exact determination, which he argued would be the result of an analytical function. Furthermore, his impression of why area was important or right came from authority-that you don't know that counting boxes is the right way "unless they tell you that's what it is or you know from previous experiences"-rather than from an understanding of the connection between the integral and the area.

In order to perform an analytical integration, Andrew first self-imposed a constraint. He proposed approximating the integrand $[E(r)]$ by an algebraic function by looking at the slope for the first linear section of the curve. Although he thought that the approximation in the second nonlinear regime would not be as simple, he still thought the approximate function would give a more accurate result than approximating the area under the curve.

\section{Case B4: Monica beyond the analytical derivation game-counting boxes to find $\Delta V$}

Soon after reading the question, Monica suggested that she needed to perform an integration to solve the problem. When the interviewer asked her why she needed to do the integration, she responded that she needed it because she was finding the potential difference between the two points. As in the temperature problem, the rate equation seemed to have triggered Monica to perceive that problem involved performing integration as seen in the following conversation:

2

3

4

5

6

7

8

9

10

11

12

13

14

15

16

17

18
1 Monica: Umm, this is what I'll do, the original
function [first writes $E(r)=-\frac{d V}{d r}$ then rearranges it as $d V=-E(r) d r]$
I: So I'm... I'm asking this thing [pointing at $d V$ ]. What's this, this thing?
Monica: Oh, that's, that's the potential differ- ence. So it would be... This is, given this graph, so you'd want to integrate over certain, over those positions, those $r \mathrm{~s}$.
I: So, is this [pointing to $\Delta V$ ] exactly the same thing as that [referring to $d V$ ] you have here?
Monica: No. This is the change in $V$ and you just...
I: Okay, what's difference between...?
Monica: Oh, never mind. Well, the difference would be the change...
I: What's difference between these two? [pointing at $d V$ and $\Delta V$.] 


$\begin{array}{ll}19 & \text { Monica: [pointing at } d V \text { ] This isn't, like, very } \\ 20 & \text { very small change in } V \text { ? That }[\Delta V \text { ] would be a } \\ 21 & \text { large, larger change in } V \text {. So, you'd have to actu- } \\ 22 & \text { ally integrate that from some } V \text { initial to } V \text { final } \\ 23 & \text { [writes } \int_{V_{i}}^{V_{f}} \text { in front of } d V \text { ]. So, then you would } \\ 24 & \text { get that [referring to } \Delta V \text { ] } \\ 25 & \text { I: Okay. So how do you get potential difference? } \\ 26 & \text { Monica: Using [pointing at } \frac{1}{4 \pi \epsilon_{0}} \frac{q}{R^{3}} r \text { ] ... You have } \\ 27 & \text { [writing } V_{f}-V_{i} \text { and inserting } \int_{0.2}^{0.8} \text { in front of pre- } \\ 28 & \text { viously written } E(r) d r \text { ] } . . \text { and if you wanted to } \\ 29 & \text { go from } .2 \text { to } .8 \text { centimeters with that function } \\ 30 & \text { [referring to } \frac{1}{4 \pi \epsilon_{0}} \frac{q}{R^{3}} r \text { ], that would be, this would } \\ 31 & \text { be, umm, the area from those two points of that } \\ 32 & \text { function, of this electric field. }\end{array}$

Monica's rearranging of the given rate equation (lines $1-3)$ and use of the operation of integration (line 8) in the above excerpt are moves in the analytical derivation game. In response to the interviewer's question about the way to get the potential difference, Monica simply integrated $E d r$ between the given limits and suggested substituting the integrand $E(r)$ by the given function $\frac{1}{4 \pi \epsilon_{0}} \frac{q}{R^{3}} r$. Monica was aware that the values of $\epsilon_{0}$ and $Q$ are not provided, but she proceeded to solve the problem analytically, indicating that her target form was a series of equations. She derived an expression for the required quantity without caring about a final numerical answer. This target form along with her moves involving equation rearranging and application of integration show that she was predominantly engaged in analytical derivation, although she briefly mentioned in the end that her integral was equivalent to the area between the given limits (lines 26-32). Monica seemed to be aware of the essence of both analytical and graphical resources for solving the problem. Although she was dominantly involved in the analytical derivation game, she briefly invoked the notion of area in the end, but she did not elaborate clearly how the area and the integral are related. In order to probe her understanding about this relationship, the interviewer asked

$\begin{array}{ll}33 & \text { I: So how do you get that [pointing at } \Delta V \text { ] in the } \\ 34 & \text { graph? } \\ 35 & \text { Monica: Umm, wait is that the fu-... This is the } \\ 36 & \text { function of that. I guess, you could integrate that. }\end{array}$

Although the interviewer asked her to explain how she would find $\Delta V$ from the graph, she focused only on the algebraic form of the integrand presented on the graph and explained how she would use that to find the required quantity analytically.

$\begin{array}{ll}37 & \text { Monica: Umm, well this } E \text { would be that [point- } \\ 38 & \text { ing at } \frac{1}{4 \pi \epsilon_{0}} \frac{q}{R^{3}} r \text { on the graph]. So you'd have } \\ 39 & \text { [mumbles and writes } \int_{0.2}^{0.8} \frac{1}{4 \pi \epsilon_{0}} \frac{q}{R^{3}} r \text { ] .. and those } \\ 40 & \begin{array}{l}\text { are all cons-. Well I'm assuming that } q \text { will be a } \\ \text { constant. }\end{array} \\ 41 & \end{array}$

(Table continued)

\begin{tabular}{ll}
42 & I: Yeah, $q$ is constant. \\
43 & Monica: Yeah, so you could bring all that out, \\
44 & [Mumbles and writes $\left.\frac{1}{4 \pi \epsilon_{0}} \frac{q}{R^{3}} \frac{r^{2}}{2}\right|_{0.2} ^{0.8}$. That would \\
45 & be..., you can calculate it. \\
46 & I: Okay. So that' s... \\
47 & Monica: ...that's the change in the electric... \\
48 & Well, that's the change in the potential difference \\
49 & [sic]. \\
50 & I: Okay. So how how else would you do that to \\
51 & find the exact number? \\
52 & Monica: You could just look at the graph and go \\
53 & from [mumbles], go from .2 to .8 and then find the \\
54 & area under the curve for those two, which would \\
55 & be... \\
56 & I: How do you find the area then? \\
57 & Monica: Umm, I guess I'd make it about a tri- \\
58 & angle... (Monica first counts the total number of \\
59 & boxes contained in the square and the triangle as \\
60 & 48 and 30, respectively. Then she adds them to \\
61 & get 58 boxes and proposes to multiply the total \\
62 & number of boxes by the value of a single box to \\
63 & get the required potential difference.) \\
\hline
\end{tabular}

In the above episode, Monica seemed to be primarily engaged in analytical derivation, and she did not look for an alternative way to find the required quantity at first. Although the problem asked for a numerical value for the potential difference, Monica simply left her answer in form of an equation, leaving behind the unknowns, such as $q$ and $\epsilon_{0}$. She seemed to be satisfied with her result that she derived from analytical derivation $\left(\left.\frac{1}{4 \pi \epsilon_{0}} \frac{q}{R^{3}} \frac{r^{2}}{2}\right|_{0.2} ^{0.8}\right)$ and considered that the resultant expression was the answer to the problem being asked. However, when the interviewer deliberately prompted her for an alternative approach, not only did she successfully show the relationship between the integral and the notion of area under the curve, but she also explained how she would evaluate the area in the given context. The interviewer's prompt for the alternative graphical approach seemed to have triggered Monica to explore additional resources, e.g., graphical. In addition to the interviewer's prompt, the incomplete result of her analytical derivation game also led her to explore alternative resources. She explored the graphical resources by playing the graphical analysis game. In this game, her target form was the given graph and the game involved analyzing it to identify one or more relevant graphical features and evaluate the required potential difference. More explicitly, her moves included identifying the area under the curve as the relevant feature and evaluating it by finding the areas of the triangle and the rectangle under the curve.

\section{DISCUSSION}

In the cases presented above, students manifested three types of e-games while solving the temperature and the electrostatics problems-recursive plug-and-chug, 
analytical derivation, and graphical analysis games. Based on these cases, there are three facets of the data to discuss. First, we make the case for analytical derivation as an epistemic game distinct from recursive plug-and-chug. Second, we point out how analytical derivation is necessary but not sufficient for solving the problems presented here. Third, we discuss the ways students went beyond analytical derivation to try an alternate solution method that may or may not have built on the result of their analytical derivation game. In addition, we also point out findings from other researchers that we see as evidence of analytical derivation in a different physics context, with different mathematical content, and discuss additional support for the idea that analytical derivation is a general epistemic game played by both experts and novices in physics. Finally, we present a brief discussion on potential instructional implications of this game.

\section{A. Analytical derivation as a distinct epistemic game}

Cases $A 1$ and $B 1$ a document the way students play the recursive plug-and-chug game, consistent with Tuminaro and Redish [9]. The epistemic form in this game involves one or more ready-made equations or expressions and the moves include plugging in numbers to evaluate the required quantity by using the given values and/or extracting them from the given graph. In the cases presented above, the epistemic forms for Alex and Kara were the generic slope expression $\frac{y_{2}-y_{1}}{x_{2}-x_{1}}$ and the given physics equation $E(r)=\frac{1}{4 \pi \epsilon_{0}} \frac{q}{R^{3}} r$, respectively. Their moves included extracting the relevant numbers from the given graphs and plugging them into their equations. Unlike in Tuminaro and Redish's study where the students used other equations for unknown quantities, students in this study used the given graphs to extract the values of unknown quantities.

All the other cases, except the above two, document the ways students play analytical derivation. Unlike the epistemic form for the recursive plug-and-chug game, the epistemic form for this game is a series of equations, which leads the students towards deriving an expression for the required quantity. Students may still proceed to plug numbers into the derived expression, but the primary purpose of the game is to use mathematically based procedural resources to derive the expression. Students generally start this game with an equation and through a series of mathematical procedures derive an expression for the required quantity in terms of known and/or unknown variables. As seen in the depicted cases, most of the students started with the rate equation $E(r)=-\frac{d V}{d r}$ and derived the expression for the required potential difference as $\Delta V=-\int_{0.2}^{0.8} \frac{1}{4 \pi \epsilon_{0}} \frac{q}{R^{3}} r d r$.

The analytical derivation game is distinct not only from recursive plug-and-chug, but also from the other games proposed by Tuminaro and Redish, including mapping mathematics to meaning and mapping meaning to mathematics. Although one may map an equation derived from analytical derivation to physical meaning or map physical meaning to acquire an expression during the analytical derivation game, the intention of analytical derivation is quite different from those of the two mapping games. The main intention of the analytical derivation game is to derive a mathematical expression using procedural resources. Unlike the other two games, the analytical derivation game may or may not involve using physics resources; often students do not use physical interpretations while deriving equations. Besides Tuminaro and Redish's games, the analytical derivation game is also distinct from Chen et al.'s answer making game. Unlike in analytical derivation, the primary focus of answer making is how one acquires an answer to a question-first answer-then-justify or first reason-thenanswer. The answer making game may or may not need a sophisticated mathematical derivation; it may only need a simple plugging-and-chugging of numbers proceeded by conceptual reasoning.

\section{B. Analytical derivation as a necessary but not sufficient epistemic game}

In order to correctly solve the temperature and electrostatics problems, students were expected (a) to recognize the desired physical quantities as definite integrals and (b) to identify the connection between the definite integral and the area under the curve. More than two-thirds of the students successfully completed part (a) by playing analytical derivation, but only one-third successfully completed both part (a) and part (b). Although the first part

TABLE I. Epistemic games played by the individual participants. RP\&C stands for recursive plug-and-chug; GA stands for graphical analysis; and AD stands for analytical derivation. A slash (/) in between two games in a column means that the subject changed games partway through the task, often when the interviewer asked for an alternate solution. Bolded rows indicate participants with excerpts in the text. Dots $(\cdots)$ indicate cases where the problem was not attempted by the participant.

\begin{tabular}{lll}
\hline \hline Subject & Temperature & Electrostatics \\
\hline Amy & $\ldots$ & RP\&C/AD \\
Alex & RP\&C & $\ldots$ \\
Andrew & GA/AD & RP\&C/AD \\
Brian & AD & AD/GA \\
Chris & GA & AD/GA \\
Jake & RP\&C/AD/GA & AD/GA \\
Joe & GA & AD/GA \\
Kara & GA & AD/GA \\
Lin & GA & AD \\
Monica & GA & AD/GA \\
Nikki & RP\&C & RP\&C \\
Ryder & RP\&C & $\cdots$ \\
Wilson & GA & GA \\
Yu & RP\&C & AD \\
\hline \hline
\end{tabular}


could be achieved simply by invoking the fundamental theorem of calculus, students often pursued an analytical approach to derive the relation. For most of the students the analytical derivation game seemed to be necessary for realizing the relationship between the required physical quantity and the definite integral. Although the problem required them to find a numerical answer, most of the students simply stopped the process after deriving the equation. At all levels of physics problem solving, students are required not only to derive equations, but also to meaningfully interpret the results of the derivations. In addition to deriving an equation, a complete solution to a problem may often demand several other actions depending upon the nature of the problem, such as examining limiting conditions, representing the equation graphically, interpreting the physical meaning of the equation, and evaluating and/or estimating a numerical value. Analytical derivation is not a sufficient game in the sense that it becomes only a routine procedure when the process does not involve any deep conceptual thinking, neither mathematical nor physical.

One indication of the breadth of use of analytical derivation can be seen when the use of various epistemic games by participant is shown (see Table I). Three participants played the analytical derivation game to deal with the temperature problem and 10 participants played this game to deal with the electrostatics problem (two of the remaining students did not engage with the electrostatics problem). There are two plausible explanations for the asymmetry in this result. First, the interview subjects were drawn from the second-semester physics course, which focuses primarily on electricity and magnetism; the electrostatics problem may have dealt with familiar content. Second, the electrostatics problem included the analytical expression for the electric field, perhaps priming analytical derivation as a reasonable game to start with, whereas the temperature problem provided the graph without any analytical expressions.

To check whether student course performance corresponded to use of epistemic games, we looked at students' grades in each of the calculus and physics sequence courses and their choice of epistemic game use for the temperature and the electrostatics problems. We found no connection between course grades and game choice: subjects at both ends of the grade spectrum in both sets of courses used analytical derivation in these two problems [31].

An important aspect of physics problem solving is the appropriate use of units. As seen above, students used a unit-based approach in their analytical derivation game (e.g., Kara in case A2); this occurred not only in the nearly linear regions, but also in the nonlinear region of the functions. This is a clear indication of students' failing to use the appropriate underlying mathematical concept- the FTC. We also found that a number of students in this study substituted values for variables in equations without any units, e.g., " 0 " and " 1 " in the integral limits in the temperature problem (see Fig. 5). Particularly, during numerical substitution, the inclusion of units provides not only a means to check for consistency in mathematical operations, but also a way to interpret the physical meaning of the result.

In the interviews, although some students were able to derive the equation for potential difference as $\Delta V=$ $-\int_{a}^{b} E d r$ and were also able to identify the connection between the definite integral and the area under the curve, they still failed to demonstrate a deeper understanding of the relationship. The students were cued by the analytically derived definite integral expression to invoke the area under the curve. But they were unable to tell how the integrals and the area under the curve were related. Only a few students manifested their understanding of the concept by invoking the notion of the Riemann sum. Similarly, the majority of the students did not manifest a deep understanding of the relation between the rate equations and the integrals - a change or accumulation of a quantity depends on the rate of change of the quantity. The lack of evidence of a deep understanding of the integral concept in the students' responses resonates well with findings from other researchers $[2,15,28,29,32,33]$.

\section{Students' exploring beyond analytical derivation}

As mentioned above, most of the students who derived equations did not initially attempt to find numerical answers. However, when the interviewer asked for numerical answers, the students either approximated the given curve with an algebraic function or assumed the curve to be too linear to continue their analytical approach. Only a few students were able to connect their previously derived equation to the relevant graphical feature, which is the expected path to achieve an optimum numerical answer. Students were directed to this path only after the intervention of the interviewer, when they were asked for either a numerical answer or an alternative approach. There were clear indications that the students possessed the knowledge base required to solve the problem, but failed to apply this knowledge in the relevant contexts. The students' failure to activate the relevant knowledge base might be attributed to their initial framing of the problems.

In case B3, Jake seemed to have all the knowledge resources needed to correctly solve the problem, but he focused mostly on the analytical derivation game. He exhibited a strong belief that the analytical derivation game was the best way to solve the problem even though it could be solved graphically by evaluating the area under the curve. He suggested that only by solving the problem analytically can he get an accurate answer. He was reluctant to evaluate the area under the curve because it would only yield an estimated answer and not an exact answer. This robust epistemological framing seemed to have held him back from pursuing the graphical analysis game. This is in 
agreement with the stance of Hammer et al. that students fail to apply their existing knowledge to a novel situation because of their framing of the situation in a specific way [25].

Similar to Tuminaro and Redish's findings, we also found that students' epistemic games were often determined by their initial framing of a problem, as seen in the above examples; thus, they were highly context dependent. During the interviews students did not only use a single strategy, but often varied their strategies (epistemic games) while solving a problem. Students switched their epistemic games, particularly, when they were confronted with varied contexts or representations and/or asked for further clarification (knowledge elicitation). We believe that students'switching of epistemic games is the consequence of shifts in their frames that are induced by the above mentioned factors. However, this conjecture can only be verified by a further deep analysis of students'epistemological framing.

\section{Analytical derivation in other contexts}

Given the structure of analytical derivation, we suggest that this game is played not only by students, but also by experts. In their work on experts'strategies for solving partial derivative problems in thermodynamics, Kustusch et al. [34] documented a set of expert activities (e.g., identifying mathematical relationships and identifying constraints) that they also established as different from the moves in the recursive plug-and-chug game. They defined this as part of their partial derivatives game. Their description of the distinction is similar to ours.

[all of the experts in their interviews] did connect [mathematical] quantities to the mathematical structure of the problem and to their conceptual understanding of the mathematics, which was fairly sophisticated and nuanced in some cases. In fact, the constraints that these experts placed on their mathematical moves, particularly in the Partial Derivatives game, clearly distinguished their activities from the Recursive Plugand-Chug game, which involves little to no evaluation of ones moves. [[34], p. 16].

This description is very similar to what we see in the analytical derivation game, but in a different mathematical context. Their move of "identifying constraints" is a form of the "assumptions or approximations" move in analytical derivation. Thus, we argue that Kustusch et al. were seeing evidence of analytical derivation, which is a larger grain-sized game than partial derivatives and can be played in any context for which the target form is an expression for a desired physical quantity. In other words, their experts were most likely playing the analytical derivation game while solving partial derivative problems. Given that it was used by experts in thermodynamics problem solving using the mathematics of partial derivatives, we suggest that this game may be seen commonly in other upper-division and graduate-level problem-solving contexts.

As mentioned in Sec. II C, Bing and Redish [11] suggest that expertlike problem solving involves a larger grainsized frame that includes frame shifting within a problem, as needed. It may be that analytical derivation is a game that includes this more expansive framing, allowing for the blending of different frames - and epistemic games - as needed. It may be that analytical derivation has an accompanying frame of its own, one of the frames among which experts shift.

We have evidence of students locked in to analytical derivation even when it did not lead to a (numerical) solution; these students did not, on their own, invoke the graphical analysis game to extract useful information to put into their derivation. This suggests that difficulties during problem solving can occur not only when a student frames a problem inappropriately [11], but also when students fail to change their epistemic game when it becomes unproductive. This supports the need for flexible use of multiple e-games.

These findings might suggest the existence of different epistemological framing for the different e-gamesdifferent expectations about what kind of knowledge is needed to solve the problem at hand-but we do not have sufficient evidence to claim the identification of one or more particular frames to accompany the analytical derivation game; that is left as a task for future research.

\section{E. Instructional implications}

The concepts of the FTC and the definite integral are widely applicable in physics problem solving. Students usually learn much of the mathematics concepts and skills required to solve the FTC-based problems in their calculus classes. However, studies show that students have difficulty applying their concepts and skills in relevant situations $[1,2,4]$. Our study shows that students do not usually lack mathematical concepts and skills needed for solving physics problems; instead, we argue that these students have difficulties accessing and implementing the relevant concepts in relevant situations. This was clearly manifested in the cases presented in Secs. IV C 1 and IV C 2. Kara revealed that she possessed all the knowledge required to correctly solve the electrostatics problem, but she was unable to find the right connections within her fragmented knowledge, until the interviewer went on probing her deeper and deeper through follow-up questions. Jake in Sec. IV C 4 initially attempted the analytical derivation game to solve the electrostatics problem. Only after the interviewer pressed for an alternative strategy, Jake pointed out the that the problem could be solved by finding the area under the curve. He clearly indicated that he could count the squares to find the required area. In response to the problems not presented here, he also showed how the definite integral and the area under the curve are related by 
invoking the Riemann sum concept. Like Jake, Joe in Sec. IV C 3 also invoked one after the other approaches including the graphical as the interviewer asked for the alternative strategies. It was apparent that he knew the right way to solve the problem, but just did not invoke it right away. From an instructional point of view, it is important to recognize the difference between these two perspectives. The former suggests that students need to be (re)taught the missing knowledge; our data do not support this approach. Instead, our findings suggest that instruction emphasize the appropriate framing of a problem, helping students decide what strategies (e-games), and thus which resources, to bring to bear.

Even for appropriate initial choice of framing and epistemic games, flexibility must be taught. Like recursive plug-and-chug, if students are playing the analytical derivation game, they may merely be invoking procedural resources pseudoconceptually, without invoking any conceptual resources. Problems for which this is a pitfall could be modified by including prompts that demand physical explanation, graphical elaboration, limiting cases evaluation, etc., in order to cue other resources and possibly the invocation of other epistemic games.

\section{F. Limitations of the study and future implications}

Because of the qualitative nature of this study, only a small number of participants were recruited from the target population, yielding a sample of convenience. In order to explore the commonality of students playing the analytical derivation game when solving physics problems, an expanded study would be needed that accessed more physics contexts, with a larger population that included different groups such as algebra-based introductory, upperdivision, and graduate physics students. Although we did not focus on the variation among the students in our population, there may be some differences in the ways the students with and without multivariable calculus solved the given problems. This study focused on a specific mathematical topic: student application of the FTC and the definite integral. In order to generalize our findings, additional studies should explore other mathematical concepts that are commonly invoked in physics problem solving, such as derivatives and partial derivatives. Similarly, this study could be expanded by observing students solving problems involving alternate representations, e.g., only numerical or only graphical. Finally, because of the narrow scope of this study, many variables such as gender, educational background, and race were not investigated.

\section{CONCLUSION}

Although much of reformed instruction de-emphasizes analytically based problem solving skills, the ability to derive equations using various mathematical resources is an important problem-solving skill required in all levels of physics. Particularly, in upper-division and graduate-level physics, problem solving often involves finding symbolic expressions for one or more physical quantities, which may or may not require a numerical answer. This mode of problem solving, i.e., analytical solutions, usually involves basic algebra and calculus operations at a minimum, and sometimes application of more sophisticated mathematical knowledge. Since the term "analytical" carries a broad meaning, we use the term analytical derivation to indicate the mode of problem solving that involves the derivation of one or more physical quantities in forms of symbolic expressions using various mathematical resources. The dominant mathematical resources students use in this type of problem solving are procedural resources such as differentiation and integration. In many instances, students use this mode of problem solving, particularly when they are provided with an equation and/or an algebraic expression.

We identified the analytical derivation game by characterizing its epistemic form, knowledge elements or resources, moves, entry and exit conditions, and constraints. The characterization of the components not only led us to identify a new e-game, but also helped us to understand the students'difficulties with the application of the fundamental theorem of calculus in physics problem solving. Students seemed to have the knowledge about all facets of the FTC required to solve the given problems, but have difficulties connecting the knowledge because of the way they frame the situations, e.g., failing to frame the problems as involving graphical interpretation of definite integrals. To some extent, we also gained insight on how a change in students'frames led them to change their epistemic games and how they apply situation-specific knowledge resources in their problem solving.

Although the students in this study were expected to identify the connection between the definite integral and the area under the curve to find a reasonable numerical answer, the majority of the students attempted the problem, at least in the beginning, using the analytical derivation game. To a larger extent, students' choice of this game seemed to be influenced by the way they initially framed the given problems. The presence of equations, algebraic expressions (functions), or even symbols seemed to lead students to frame the problems as requiring equation derivation, thus invoking the analytical derivation game. Some students seemed to choose this game over other games in order to get a better numerical answer. They chose this game with the goal of obtaining the most exact numerical answer, even if the resulting equation had one or more unknown variables.

Problem solving in physics often requires interpretation and/or application of one or more types of representations, such as algebraic, graphical, and numerical. The algebraic representation mostly involves symbolic manipulations and equation derivations, which may require skills ranging 
from very basic to highly sophisticated mathematical procedures and recognition of appropriate constraints and assumptions about the physical situation. Students tend to employ an algebraic representation to derive one or more equations even when a problem does not require any equation derivation. There may be more than one reason for the students' excessive inclination towards playing with equations while solving a problem. Much of physics problem solving, particularly in traditional instruction, is dominantly algorithmic or rule based, which encourages symbolic manipulation and so-called equation chasing. Students are often reluctant to use other representations because the symbolic manipulation and the equation derivation could be processed routinely without engaging much in a deep thinking process. This is true not only in physics but also in mathematics problem solving. Selden et al. reported that even above-average calculus students often struggle while solving nonroutine problems that do not involve symbolic manipulation, tending to solve these calculus problems simply algebraically [35,36]. Previous studies have documented that students have difficulties switching between and connecting two or more representations, including algebraic and graphical $[37,38]$. This difficulty often leads them to pursue solely the algebraic representation and avoid other representations such as graphical.

\section{ACKNOWLEDGMENTS}

The authors wish to acknowledge members of the UMaine Physics Education Research Laboratory for their feedback and suggestions. This work is supported in part by the National Science Foundation through Grants No. DUE0817282 and No. PHY-1405726.
[1] L. Cui, N. S. Rebello, P. Fletcher, and A. Bennett, Transfer of learning from college calculus to physics courses, in Proceedings of the National Association for Research in Science Teaching (NARST), San Francisco (2006).

[2] D. Nguyen and N. S. Rebello, Students' understanding and application of the area under the curve concept in physics problems, Phys. Rev. ST Phys. Educ. Res. 7, 010112 (2011).

[3] D. Hu and N. S. Rebello, Understanding student use of differentials in physics integration problems, Phys. Rev. ST Phys. Educ. Res. 9, 020108 (2013).

[4] F. R. Yeatts and J. R. Hundhausen, Calculus and physics: Challenges at the interface, Am. J. Phys. 60, 716 (1992).

[5] E. F. Redish, arXiv:physics/0608268.

[6] J. F. Wagner, C. A. Manogue, and J. R. Thompson, Representation issues: Using mathematics in upper-division physics, AIP Conf. Proc. 1413, 89 (2012).

[7] M. T. Chi, P. J. Feltovich, and R. Glaser, Categorization and representation of physics problems by experts and novices, Cogn. Sci. 5, 121 (1981).

[8] P. B. Kohl and N. D. Finkelstein, Student representational competence and self-assessment when solving physics problems, Phys. Rev. ST Phys. Educ. Res. 1, 010104 (2005).

[9] J. Tuminaro and E. F. Redish, Elements of a cognitive model of physics problem solving: Epistemic games, Phys. Rev. ST Phys. Educ. Res. 3, 020101 (2007).

[10] B. R. Wilcox, M.D. Caballero, D. A. Rehn, and S. J. Pollock, Analytic framework for students' use of mathematics in upper-division physics, Phys. Rev. ST Phys. Educ. Res. 9, 020119 (2013).

[11] T. Bing and E. Redish, Analyzing problem solving using math in physics: Epistemological framing via warrants, Phys. Rev. ST Phys. Educ. Res. 5, 020108 (2009).
[12] D. E. Meltzer, The relationship between mathematics preparation and conceptual learning gains in physics: A possible, "hidden variable", in diagnostic pretest scores, Am. J. Phys. 70, 1259 (2002).

[13] E. B. Pollock, J. R. Thompson, and D. B. Mountcastle, Student understanding of the physics and mathematics of process variables in $P-V$ diagrams, in AIP Conf. Proc. 951, 168 (2007).

[14] P. W. Thompson, Images of rate and operational understanding of the Fundamental Theorem of Calculus, Educ. Studies Math. 26, 229 (1994).

[15] P. W. Thompson and J. Silverman, The concept of accumulation in calculus, in Making the Connection: Research and Teaching in Undergraduate Mathematics Education, edited by M. P. Carlson and C. Rasmussen (Mathematical Association of America, Washington D.C., 2008), Vol. 73, pp. 43-52.

[16] R. J. Beichner, Testing student interpretation of kinematics graphs, Am. J. Phys. 62, 750 (1994).

[17] L. C. McDermott, M. L. Rosenquist, and E. H. Van Zee, Student difficulties in connecting graphs and physics: Examples from kinematics, Am. J. Phys. 55, 503 (1987).

[18] R. R. Bajracharya, T. M. Wemyss, and J. R. Thompson, Student interpretation of the signs of definite integrals using graphical representations, AIP Conf. Proc. 1413, 111 (2012).

[19] R. R. Bajracharya and J. R. Thompson, Student application and understanding of the fundamental theorem of calculus at the mathematics-physics interface, in Proceedings of the 17th Annual Conference on Research in Undergraduate Mathematics Education, XVII, edited by T. FukawaConnolly, G. Karakok, K. Keene, and M. Zandieh (Denver, 2014), pp. 394-400. 
[20] A. Collins and W. Ferguson, Epistemic forms and epistemic games: Structures and strategies to guide inquiry, Educ. Psychol. 28, 25 (1993).

[21] S. Vinner, The pseudo-conceptual and the pseudoanalytical thought processes in mathematics learning, Educ. Studies Math. 34, 97 (1997).

[22] S. Rasslan and S. Vinner, Images and definitions for the concept of even/odd function, in Proceedings of the 21st Conference of the International Group for the Psychology of Mathematics Education (Lahti, Finland, 1997), Vol. 4, pp. $41-48$.

[23] E. Gire, D. Nguyen, and N. S. Rebello, Characterizing students' use of graphs in introductory physics with a graphical analysis epistemic game, in NARST Annual Meeting (Orlando, FL, 2011).

[24] Y. Chen, P. W. Irving, and E. C. Sayre, Epistemic game for answer making in learning about hydrostatics, Phys. Rev. ST Phys. Educ. Res. 9, 010108 (2013).

[25] D. Hammer, A. Elby, R. E. Scherr, and E. F. Redish, Resources, framing, and transfer, Transfer of Learning from a Modern Multidisciplinary Perspective, edited by $\mathrm{P}$. Mestre (Information Age Publishing, Greenwich, 2005), pp. 89-119.

[26] P. Hutchison and D. Hammer, Attending to student epistemological framing in a science classroom, Sci. Educ. 94, 506 (2010).

[27] J. Tuminaro, Ph.D. thesis, University of Maryland, College Park, MD, 2004.

[28] T. A. Grundmeier, J. Hansen, and E. Sousa, An exploration of definition and procedural fluency in integral calculus, PRIMUS: Problems, Resources, and Issues in Mathematics Undergraduate Studies 16, 178 (2006).

[29] V. Sealey, Definite integrals, Riemann sums, and area under a curve: What is necessary and sufficient, in Proceedings of the 28th Annual Meeting of the North American Chapter of the International Group for the
Psychology of Mathematics Education (Merida, Mexico, 2006).

[30] A. Strauss and J. M. Corbin, Grounded Theory in Practice (SAGE Publications, Thousand Oaks, CA, 1997).

[31] In particular, Brian earned A's in all five courses; Andrew earned grades from A to B+ in calculus and A's in physics; and Jake's grades were $\mathrm{B}+$ to $\mathrm{C}$ in calculus and $\mathrm{C}+$ and $\mathrm{D}+$ in physics.

[32] M. P. Carlson, J. Persson, and N. Smith, Developing and connecting calculus students' notions of rate-of change and accumulation: The Fundamental Theorem of Calculus, Proceedings of the 2003 Joint Meeting of PME and $P M E-N A$, edited by N. A. Pateman, B. J. Dougherty, and J. T. Zillion (University of Hawaii, Honolulu, 2003), Vol. 2, pp. $165-172$.

[33] A. Orton, Students' understanding of integration, Educ. Stud. Math. 14, 1 (1983).

[34] M. B. Kustusch, D. Roundy, T. Dray, and C. A. Manogue, Partial derivative games in thermodynamics: A cognitive task analysis, Phys. Rev. ST Phys. Educ. Res. 10, 010101 (2014).

[35] J. Selden, A. Mason, and A. Selden, Can average calculus students solve nonroutine problems, J. Math. Behav. 8, 45 (1989).

[36] A. Selden, J. Selden, and A. Mason, Even good calculus students can't solve nonroutine problems, in Research Issues in Undergraduate Mathematics Learning Preliminary Analyses and Results, edited by J. J. Kaput and E. Dubinsky (Mathematical Association of America, Oberlin, OH, 1994), pp. 19-26.

[37] P. B. Kohl and N. D. Finkelstein, Effects of representation on students solving physics problems: A fine-grained characterization, Phys. Rev. ST Phys. Educ. Res. 2, 010106 (2006).

[38] B. Ibrahim and N. S. Rebello, Representational task formats and problem solving strategies in kinematics and work, Phys. Rev. ST Phys. Educ. Res. 8, 010126 (2012). 